\title{
Analytic continuation of the critical line in 2-color QCD at nonzero temperature and density
}

\section{Paolo Cea}

Dipartimento di Fisica, Università di Bari, and INFN - Sezione di Bari, I-70126 Bari, Italy

E-mail: paolo.cea@ba.infn. it

\section{Leonardo Cosmai}

INFN - Sezione di Bari, I-70126 Bari, Italy

E-mail: leonardo.cosmai@ba.infn.it

\section{Massimo D'Elia}

Dipartimento di Fisica, Università di Genova, and INFN - Sezione di Genova,

I-16146 Genova, Italy

E-mail: Massimo.Delia@ge.infn.it

\section{Alessandro Papa*}

Dipartimento di Fisica, Università della Calabria, and INFN - Gruppo Collegato di Cosenza, I-87036 Rende, Italy

E-mail: papa@cs.infn.it

We determine the pseudo-critical line in the temperature - chemical potential plane of 2-color QCD by direct Monte Carlo simulations and by analytic continuation from imaginary chemical potential.

The XXV International Symposium on Lattice Field Theory

July 30-4 August 2007

Regensburg, Germany

${ }^{*}$ Speaker. 


\section{Introduction}

It is widely accepted that QCD undergoes a transition from the hadronic to the so-called "quark-gluon plasma" phase for large enough temperature, depending on the baryon chemical potential. Determining the shape and the nature of this transition (or pseudo-critical) line in the temperature - chemical potential plane is of central interest in cosmology, in astrophysics and in the phenomenology of heavy ion collisions. The lattice approach, which is the natural tool to face a non-perturbative problem like this, is plagued, however, by the well-known "sign problem": for non-zero chemical potential the QCD fermion determinant becomes complex and the standard Monte Carlo importance sampling is unfeasible.

Several strategies have been invented to circumvent this problem (for a review, see [1] and [2]). Here, we concentrate on one of these approaches, the method of analytic continuation, first used in Refs. [3] and [4]. The idea behind this method is very simple: numerical simulations are performed at imaginary chemical potential, $\mu=i \mu_{I}$, for which the fermion determinant is real, then Monte Carlo determinations are interpolated by a suitable function and finally this function is analytically continued to real values of $\mu$. This method is rather powerful since temperature and chemical potential can be varied independently and there is no limitation from increasing lattice size, as in methods based on reweighting. There is, however, an important drawback: the periodicity of the partition function and the presence of non-analyticities arising for imaginary values of the chemical potential [5] limit the region useful for numerical determinations to the strip $0 \leq \mu_{I} / T<\pi / 3$. This implies that the accuracy in the interpolation of the results at imaginary chemical potential has a strong impact on the extension of the domain of real $\mu$ values reachable after analytic continuation.

Although the method is designed to infer the behavior of an observable with the real chemical potential from the knowledge of its dependence on the imaginary chemical potential, the idea to analytically continue the pseudo-critical line itself has been extensively applied $[6,7,8]$ (see Ref. [6] for a discussion on the reliability of this application of the method).

A control on the systematics of the method of analytic continuation and possible insights for its improvement can be achieved by testing it in theories which do not suffer the sign problem, by direct comparison of the analytic continuation with Monte Carlo results obtained directly at real $\mu$ [4, 9, 10, 11]. In Ref. [11], in particular, a high-precision numerical analysis in SU(2) (or 2color QCD) with $n_{f}=8$ degenerate staggered fermions has shown that, for temperatures above the pseudo-critical one at zero chemical potential, the extrapolation to real $\mu$ improves considerably if ratio of polynomials are used instead of simple polynomials in interpolating the behavior with imaginary $\mu$ of some test observables, this validating a proposal formulated in Ref. [12].

In this work we extend the numerical analysis of Ref. [11] to the study of the analytic continuation of the pseudo-critical line. The strategy is the following:

- for several fixed values of the chemical potential, both real and imaginary, we determine the (pseudo-)critical $\beta$ 's by looking for peaks in the susceptibilities of a given observable;

- we interpolate the determinations of the critical $\beta$ 's at imaginary chemical potential with an analytic function of $\mu$, to be then extrapolated to real chemical potential;

- we compare the extrapolation with the determinations of the critical $\beta$ 's at real chemical potential. 

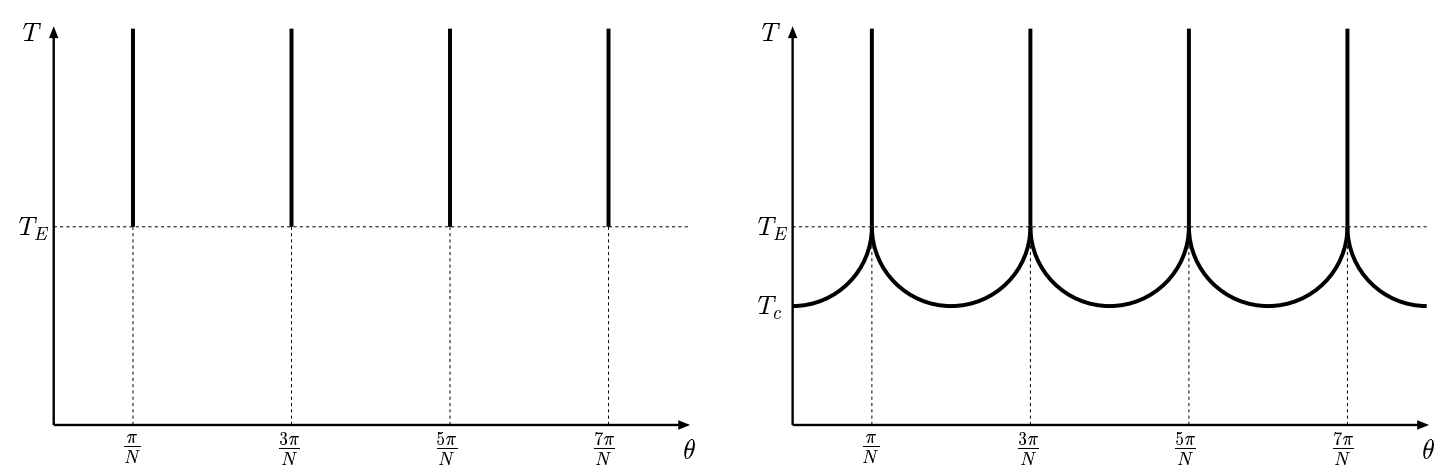

Figure 1: (Left) Phase diagram in the $(T, \theta)$ plane according to Ref. [5]. (Right) Tentative phase diagram in the $(T, \theta)$ plane after the inclusion of the chiral pseudo-critical lines.

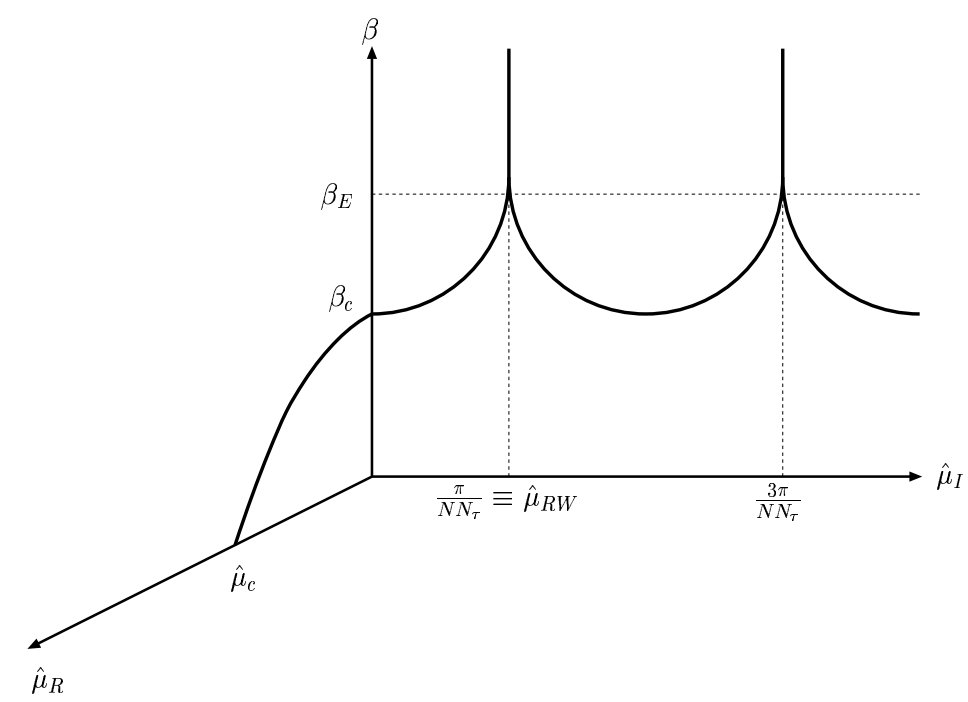

Figure 2: Phase diagram in the $\left(\beta, \hat{\mu}_{I}, \hat{\mu}_{R}\right)$-space; $N$ is the number of colors, $N_{\tau}$ the extension of the lattice in the temporal direction.

The aim of this investigation is to verify the possibility to analytically continue the pseudo-critical line and, in affirmative case, to optimize the choice of the interpolating function to be used. For a better control of the systematics, we have repeated the outlined strategy for three different observables (chiral condensate, Polyakov loop, plaquette).

\section{Theoretical background}

Long ago Roberge and Weiss have shown [5] that the partition function of any $\mathrm{SU}(N)$ gauge theory with non-zero temperature and imaginary chemical potential, $\mu=i \mu_{I}$, is periodic in $\theta \equiv$ $\mu_{I} / T$ with period $2 \pi / N$ and that the free energy $F$ is a regular function of $\theta$ for $T<T_{E}$, while it is discontinuous at $\theta=2 \pi(k+1 / 2) / N, k=0,1,2, \ldots$, for $T>T_{E}$, where $T_{E}$ is a characteristic temperature, depending on the theory. The resulting phase diagram in the $(T, \theta)$-plane is given in Fig. 1 

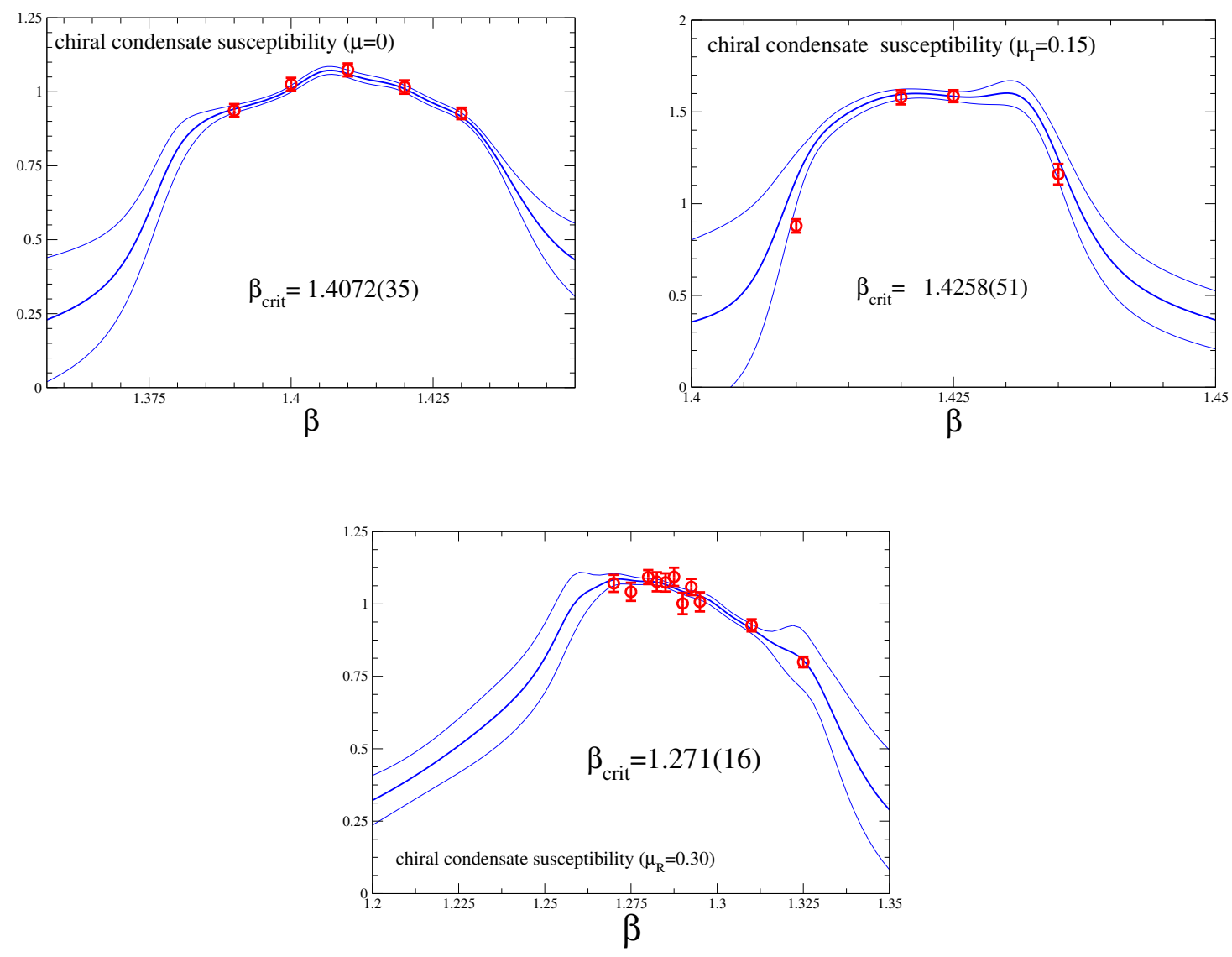

Figure 3: Susceptibility of the chiral condensate at $\hat{\mu}=0, \hat{\mu}=0.15 i$ and $\hat{\mu}=0.30$. The blue solid lines represent the result (ant the related error) of the multi-histogram reweighting.

(left), where the vertical lines represent first order transition lines. This structure is compatible with the $\mu \rightarrow-\mu$ symmetry, related with CP invariance, and with the Roberge-Weiss periodicity. The $\mu_{I}$-dependence of any observable is completely determined if this observable is known in the strip $0 \leq \theta<\pi / N$. These predictions have been confirmed numerically in several cases, studying the behaviour of quantities like the Polyakov loop and the chiral condensate [6, 8, 9].

A phase diagram like that in Fig. 1 (left) would imply the absence of any transition along the $T$ axis in the physical regime of zero chemical potential for any value of $N$, of $n_{f}$ and of the quark masses, which cannot be true. Therefore, it is necessary to admit that the phase diagram in the $(T, \theta)$-plane is more complicated than in Fig. 1 (left). The simplest possibility is given in Fig. 1 (right), where the added lines generally represent transitions which can be first order, second order or crossover. The temperature $T_{c}$ is the pseudo-critical one for the transition at zero chemical potential. In Fig. 2 the phase diagram of Fig. 1 (right) has been redrawn the $\left(\beta, \hat{\mu}_{I}\right)$-plane, where $\beta=2 N / g^{2}, \hat{\mu}_{I}=a \mu_{I}$ is the imaginary chemical potential in lattice units and it has been used the fact that $T=1 /\left(a N_{\tau}\right)$, with $N_{\tau}$ the temporal extension of the lattice. In Fig. 2 also the $\mu_{R}$-axis has been included, with a sketch of the continuation of the pseudo-critical line on the $\left(\beta, \hat{\mu}_{R}\right)$-plane. 
Table 1: $\beta_{\text {crit }}$ as determined from the peak of the susceptibilities of chiral condensate, Polyakov loop and plaquette.

\begin{tabular}{llll}
\hline \hline$\hat{\mu}$ & chiral condensate & Polyakov loop & plaquette \\
\hline $0.30 i$ & $1.5097(33)$ & $1.5023(60)$ & $1.5048(62)$ \\
$0.20 i$ & $1.4548(42)$ & $1.4355(47)$ & $1.4476(94)$ \\
$0.15 i$ & $1.4258(51)$ & $1.441(23)$ & $1.418(10)$ \\
$0.10 i$ & $1.4228(55)$ & $1.4089(63)$ & $1.415(10)$ \\
0. & $1.4072(35)$ & $1.394(17)$ & $1.4060(45)$ \\
0.20 & $1.3551(91)$ & $1.352(13)$ & $1.356(12)$ \\
0.30 & $1.271(16)$ & $1.267(26)$ & $1.286(15)$ \\
\hline \hline
\end{tabular}

Table 2: Parameters of the fit with a polynomial $A+B \hat{\mu}^{2}$ of the data at imaginary chemical potential; the last column gives the extrapolation of the pseudo-critical line at the imaginary chemical potential corresponding to the first RW transition line.

\begin{tabular}{lllll}
\hline \hline observable & $A$ & $B$ & $\chi^{2} /$ d.o.f. & $\beta_{\text {crit }}\left(\hat{\mu}_{R W}\right)$ \\
\hline chiral condensate & $1.4071(27)$ & $-1.140(50)$ & 0.91 & $1.5829(81)$ \\
Polyakov loop & $1.3931(55)$ & $-1.18(10)$ & 0.85 & $1.575(17)$ \\
plaquette & $1.4042(39)$ & $-1.104(83)$ & 0.48 & $1.574(13)$ \\
\hline \hline
\end{tabular}

\section{Numerical results}

We performed numerical simulations on a $16^{3} \times 4$ lattice of the SU(2) gauge theory with $n_{f}=8$ degenerate staggered fermions having mass $a m=0.07$, by means of a Hybrid Monte Carlo algorithm with $d t=0.01$. Simulations have been performed on the APEmille crate in Bari and on the computer facilities at the INFN apeNEXT Computing Center in Rome.

The observables we have considered are the chiral condensate, the Polyakov loop and the plaquette; for each of them we have looked for the peak in the susceptibility for varying $\beta$, at some fixed values of the chemical potential, both real and imaginary. For each observable and for each fixed value of $\hat{\mu}$, we have taken a few data points for different $\beta$ values (statistics $\sim 20000$ ) and smoothed out the susceptibility by the multi-histogram extension of the Ferrenberg-Swendsen reweighting method [13]. The uncertainty on the position of the peaks has been evaluated by the bootstrap method.

In Table 1 we summarize our preliminary results for the critical $\beta$ at each value of the chemical potential we considered. In Fig. 3 we show for illustration purposes the susceptibility of the chiral condensate at $\hat{\mu}=0.15 i, \hat{\mu}=0$. and $\hat{\mu}=0.30$.

We have then looked for an interpolation of the data of $\beta_{\text {crit }}$ for imaginary and zero chemical potential (i.e. for the first 5 entries in Table 1), and have repeated this procedure for each of the three observables considered. In all cases we have found that the optimal interpolating function is a polynomial of the form $A+B \hat{\mu}^{2}$. If different functions are used, such as larger order polynomials or ratio of polynomials, the fit puts to values compatible with zero all parameters except two of them, so to reduce the interpolating function to a first order polynomial in $\hat{\mu}^{2}$. The fit results are summarized in Table 2. We can see that the resulting parameters have a tiny dependence on 

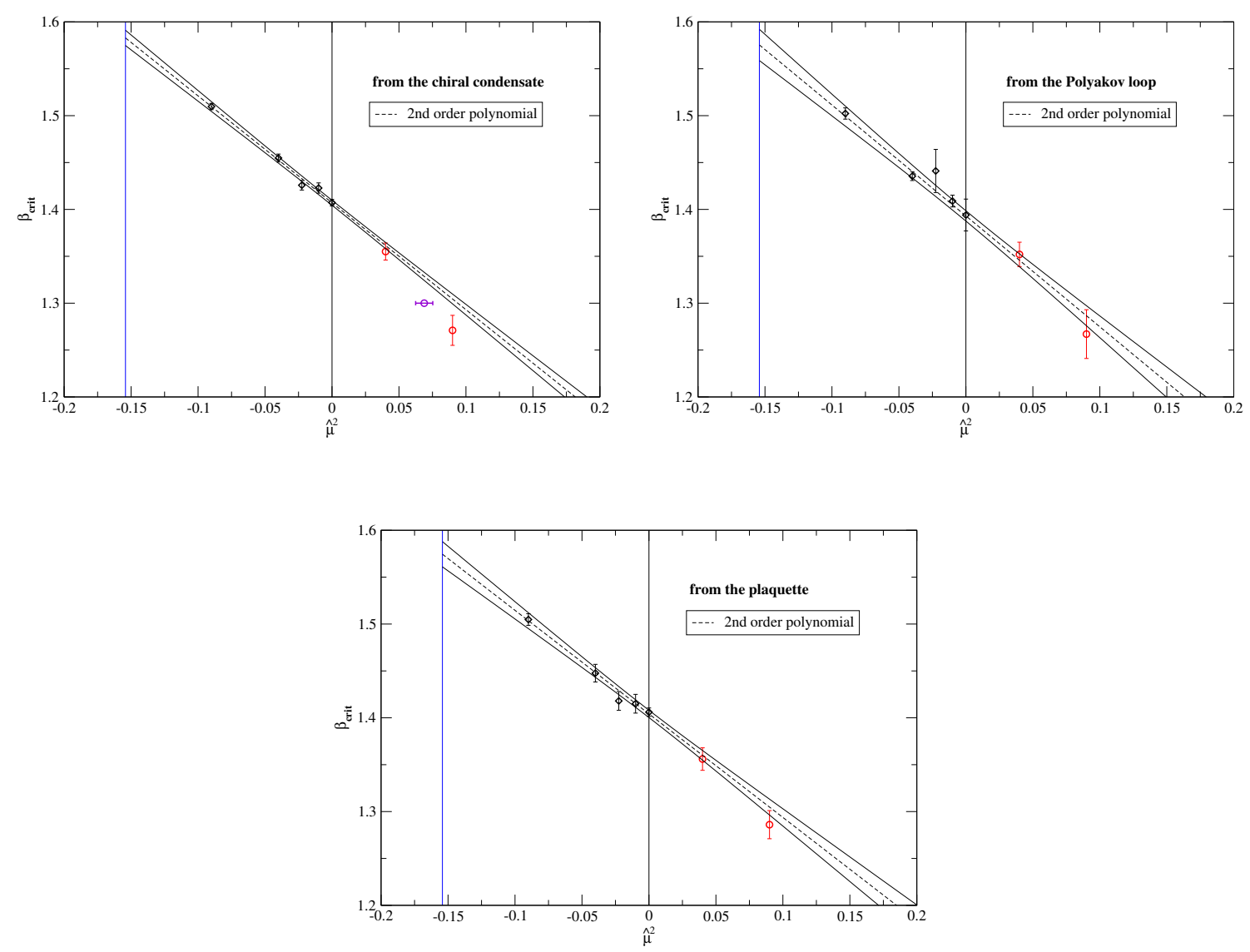

Figure 4: $\beta_{\text {crit }} v s . \hat{\mu}^{2}$ determined from the susceptibility of chiral condensate, Polyakov loop and plaquette. The dashed line is the result of a fit with a polynomial $A+B \hat{\mu}^{2}$ of the data at imaginary chemical potential $\left(\hat{\mu}^{2}<0\right)$; the black solid lines enclose the boundary of the uncertainty. The blue vertical line gives the position of the first RW transition line. The point in magenta comes from a determination of the peak of the susceptibility at $\beta=1.30$, taken from Ref. [11].

the observable considered; moreover, the extrapolation of the pseudo-critical line at the $\hat{\mu}$ value corresponding to the first Roberge-Weiss transition line, $\hat{\mu}_{R W}=i \pi / 8$, is in good agreement with an independent determination of the endpoint $\beta_{E}$ [14]. This is a confirmation of the structure of the phase diagram as sketched in Fig. 1 (right).

The most important point of the present analysis is to test whether the extrapolation of the pseudo-critical line to real $\hat{\mu}$ agrees or not with the two direct determinations of $\beta_{\text {crit }}$ available so far at real $\hat{\mu}$, i.e. $\hat{\mu}=0.20$ and 0.30 . Such comparison is presented in Figs. 4 for each of the observables considered. There is an overall substantial agreement; in the case of the chiral condensate there might be a deviation at $\hat{\mu}=0.30$, which calls for a refinement of the numerical analysis.

\section{Conclusions and outlook}

We have presented preliminary results aimed at studying the possibility of the analytic continuation of the pseudo-critical line from imaginary to real chemical potential in 2-color QCD. 
This theory is exempt of the sign problem and, therefore, makes possible to compare analytic continuations from imaginary to real chemical potential with direct determinations at real chemical potential.

By determining the position of the peaks in the susceptibilities of three observables (chiral condensate, Polyakov loop, plaquette) for varying the temperature at fixed imaginary chemical potential, we have interpolated the pseudo-critical line in the temperature - imaginary chemical potential plane. It turns out that the best interpolation for $\beta_{\text {crit }}\left(\mu^{2}\right)$ is a first order polynomial in $\mu^{2}$. The extrapolation to real chemical potential of this curve generally agrees with the direct determinations at real chemical available so far. A larger statistics and an extension of the data set could reveal possible deviations.

\section{References}

[1] O. Philipsen, PoS LAT2005 (2006) 16 [hep-lat / 0510077 ].

[2] C. Schmidt, hep-lat/0610116.

[3] M.P. Lombardo, Nucl. Phys. (Proc. Suppl.) 83 (2000) 375 [hep-lat/ 9908006 ].

[4] A. Hart, M. Laine and O. Philipsen, Phys. Lett. B505 (2001) 141 [hep-lat/ 0010008 ].

[5] A. Roberge and N. Weiss, Nucl. Phys. B275 (1986) 734.

[6] P. de Forcrand and O. Philipsen, Nucl. Phys. B642 (2002) 290 [hep-lat/ 0205016 ]; Nucl. Phys. (Proc. Suppl.) 119 (2003) 535 [hep-ph / 0301209 ].

[7] P. de Forcrand and O. Philipsen, Nucl. Phys. B673 (2003) 170 [hep-lat/ 030702 0]; Nucl. Phys. (Proc. Suppl.) 129 (2004) 521 [hep-lat/0309109].

[8] M. D'Elia and M.P. Lombardo, hep-lat / 0205022 ; Phys. Rev. D67 (2003) 014505 [hep-lat / 0209146 ]; Nucl. Phys. (Proc. Suppl.) 129 (2004) 536 [hep-lat/ 0309114 ].

[9] P. Giudice and A. Papa, Phys. Rev. D69 (2004) 094509 [hep-lat / 0401024 ].

[10] S. Kim, Ph. de Forcrand, S. Kratochvila, T. Takaishi, PoS LAT2005 (2006) 166 [hep-lat/0510069].

[11] P. Cea, L. Cosmai, M. D'Elia and A. Papa, JHEP02(2007)066 [hep-lat / 0612018]; PoS LAT2006 (2006) 143 [hep-lat/ 0610088 ].

[12] M.P. Lombardo, PoS LAT2005 (2006) 168 [hep-lat/ 0509181$].$

[13] A.M. Ferrenberg and R.H. Swendsen, Phys. Rev. Lett. 61 (1988) 2635.

[14] G. Cortese, laurea thesis, Università della Calabria (2007). 